\title{
Evaluation and Improvement Strategy of Street Space Quality in Lujiazui Core Area of Shanghai Based on Multi-source Data Fusion
}

\author{
Jiaxin Li, Urban and Rural Planning Department, School of Architecture, Southeast \\ University,NanJing,China
}

\begin{abstract}
Facing the demand of urban fine design, it has become a consensus to promote people-oriented and quality-oriented urban construction. How to improve the vitality of urban space has become the theme of urban construction. As an important factor affecting the urban environment, street space largely determines the level of urban vitality and the prosperity of urban public living space. It is extremely urgent to improve the quality of street space. In the current implementation and evaluation of urban design, there is an urgent need for a comprehensive and rapid evaluation system to evaluate the quality of street space. This paper selects the street space of Shanghai Lujiazui CBD as research object, synthesizes multi-source data to build a street space quality evaluation system including 5 indicators and 18 evaluation factors: street space carrying capacity, street space vitality, street environment comfort, street travel safety and crowd social interaction. According to the evaluation results and problems, this paper puts forward promotion strategies from three aspects: street public space, street public facilities and street support system, hoping to provide reference for the evaluation of street space construction.
\end{abstract}

\section{Keywords}

Street network, Space quality, Multi source data, Vitality improvement, Shanghai Lujiazui CBD

\section{Introduction}

Since the promulgation and implementation of a series of macro policies represented by China's National New Urbanization Plan (2014-2020), promoting people-oriented and quality-oriented urban construction has become a consensus. China's urban construction has transformed to a historical stage of fine adjustment and improvement. This means that the focus of urban planning and construction has begun to change from the concept of speed priority to the pursuit of quality, and gradually pay attention to the life comfort and happiness of urban people. Urban space has changed from incremental planning to stock planning. How to improve the vitality of urban space has become the theme of urban construction, and street plays a key role in urban public space. It is not only the window of urban appearance, but also the epitome of urban spatial form, and an important place to enhance urban vitality. As an important factor affecting the urban environment, street space largely determines the level of urban vitality and the prosperity of urban public living space, which has always been the research hotspot of scholars at home and abroad.

However, in the past aggressive urban construction, the street space is more and more inclined to the traffic attribute and ignores the humanized experience, resulting in the imbalance of street space scale, 
confusion of structure and loss of vitality. Facing the demand of urban fine design, it is extremely urgent to improve the quality of street space [1]. Before looking for improvement measures, it is first necessary to evaluate its space quality and find the short board to suit the remedy to the case. Therefore, in the current implementation and evaluation of urban design, there is an urgent need for a comprehensive and rapid evaluation system to evaluate the quality of street space.

With the advent of the information age, it brings new ideas and development opportunities for the evaluation method of street space quality [2]. In the past, only relying on the traditional data integration and drawing design can not meet the needs of a large number of urban streets. Relying on the rapid development of computer performance and convenient network platform, digital technology makes it possible to collect urban street data and conduct detailed analysis [3]. Multidimensional and multi angle data sources and sufficient data samples have entered the research horizon. By building a corresponding big data analysis platform and using open multi-source big data such as POI data, road network data, spatial morphology data, social media data and urban street view images to study urban street space and carry out vitality perception and spatial optimization has become a new direction of urban street research [4].

based on it, this paper selects the street space of Shanghai Lujiazui CBD as research object, synthesizes multi-source data to build a street space quality evaluation system including five indicators: street space carrying capacity, street space vitality, street environment comfort, street travel safety and crowd social interaction, then puts forward the promotion strategy, hoping to provide reference for street space quality evaluation work.

\section{Literature Review and Analytical Framework}

\subsection{Literature Review}

Thinking about the quality of street space is not new. The pioneers represented by Jacobs and Lefebvre began to discuss space quality and its social and economic effects as early as the 1960s [5]. Gehl, Whyte, Lynch and others have deeply studied the characteristics of urban space and constantly summarized the methods to improve the quality and vitality of urban space, with street space as the research focus [6] [9]. China's research on the quality of street space also starts from the quality of public space. Guo enzhang (1998) was the first to make a qualitative standard for the quality of urban public space [7]. He proposed that high-quality urban public space should have ten characteristics, such as sociality, identification, comfort, safety, harmony, accessibility, pleasure, ecology, culture and diversity. Street space is a kind of public space. Street space quality generally refers to two aspects: the composition of street material elements (objective) and the perception of environmental landscape quality level (subjective). Research on relevant aspects is also mainly carried out from these two aspects. For example, Hou Yan (2016) proposed that the core of space quality improvement is to improve the material elements of the street. According to the case of the space quality of streets and lanes in the old urban area of Tonglu, he puts forward countermeasures and suggestions to improve the space quality of streets and lanes in the old urban area [8]; Gan Luoshu (2018), starting from morphology and behavior, summarized the strategies and techniques for creating spatial quality of streets and lanes in Chengdu by combining the "Small Streets" project of Chengdu [10].

These research results still have guiding significance for the current research, but due to the lack of quantitative basic data and the construction of evaluation indicators, it is not conducive to further assist in refined design practice and control. With the development of the information age and the development of computer technology, the acquisition and utilization of multi-source urban data are realized. More and more street space quality evaluation methods using data calculation and quantitative analysis appear, which can be divided into the following categories: evaluation based on street view 
image data and image recognition technology, evaluation based on geospatial data and location service data, Semantic recognition method evaluation based on deep learning, spatial syntax technology evaluation, remote sensing information technology evaluation, etc. Cao Kaizhong (2011) believes that spatial syntax can effectively reveal the organizational relationship of urban street space in the category of urban research [11]; Rundle et al. (2011) studied 37 pedestrian blocks in New York by evaluating the material space index of block environment through street view pictures [13]; Sarkar (2015) and others used a wide range of travel demand survey data and vegetation cover index (NDVI) obtained from remote sensing infrared images, combined with SDNA software modeling to verify street connectivity, street vegetation density and other factors [14]; Xin Ping (2017) constructed the public space quality evaluation system of Beijing historical blocks based on the PSPL method, then evaluated the street space of Nanluoguxiang to verify the operability and implementation of the system; Fang Yonghua (2018) measured the street vitality of the main urban area of Nanjing in a wider range in combination with mobile phone signaling data and map POI data [15]; Naik (2018) and others used the data of Place Pulse project as the training set to deeply study the street view pictures of 21 cities in the United States, measure the perceived security of street space, and quantitatively analyze the security and other elements of street space [12].

It can be found that the research on street space quality should start from a comprehensive perspective, and build an urban street space quality evaluation system from two aspects: objective analysis and subjective perception. At the same time, the research of street spatial quality needs: (1) continuous, large-scale and high-precision street spatial feature measurement, rather than a few street sections; (2) The automatic evaluation model of street spatial characteristics and place quality is used to realize the rapid and accurate evaluation of large-scale data [16]. Only by solving the above two problems can we really help the practice of street space quality guidance and control.

\subsection{Analytical Framework}

Based on the above research and analysis, combined with the street space quality evaluation methods proposed by many scholars, this paper establishes a street space quality evaluation system based on multi-source data fusion. It uses multi-source data such as POI data, road network data, spatial morphology data, social media data and street view images to establish the basic database for quantitative evaluation of street space quality. The evaluation index system of street space quality was constructed by using spatial syntax analysis, panoramic segmentation analysis of street view images, functional clustering analysis, $\mathrm{POI}$ density mixing degree and heat analysis, spatial and temporal variation of population density distribution and other analysis methods. The index system relies on the two dimensions of material space composition and subjective space perception of urban street space quality, including five indicators, 18 evaluation factors in total: street space carrying capacity (construction intensity, space form, street green coverage rate, street height width ratio, street space identifiability), street space vitality (street function, street scene type analysis, street facade color analysis, business hours facing the street analysis), street environment comfort (walking convenience, landscape beauty, facilities perfection), street travel safety (street brightness, vehicle interference index, completeness of marking facilities), crowd social interaction (Baidu heat map, crowd concentration, social interface index). 


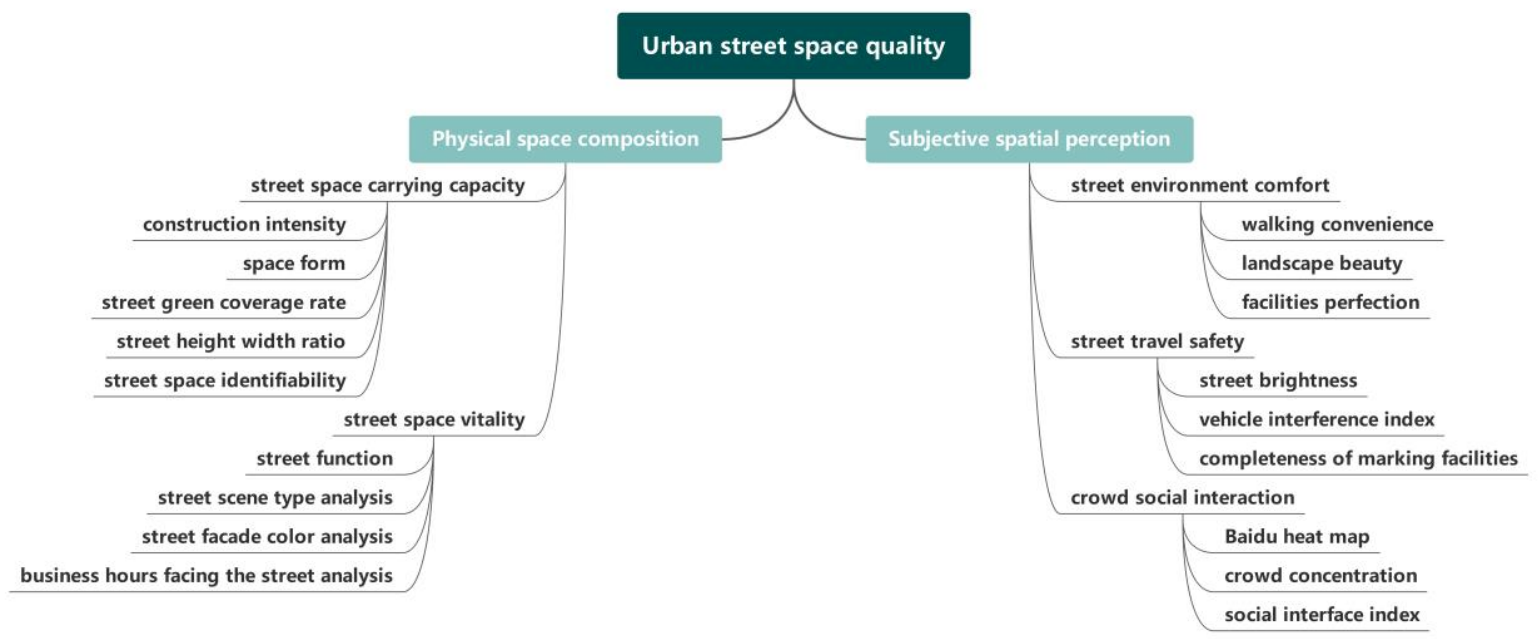

Figure 1. Evaluation index system of street space quality. Source: Author.

\section{Study Area, Data, and Methods}

\subsection{Study Area}

This paper selects the street space in the core area of Lujiazui in Shanghai, which is crowded with people and vehicles, as the research object. It is located in the eastern part of the central activity area of Shanghai, in the central area of Lujiazui financial and Trade Zone in Pudong New Area, and to the north of Lujiazui central green space. The study area is adjacent to Lujiazui Station of Rail Transit Line 2. The construction of Pudong South Road Station of rail transit line 14 in the East (the intersection of Pudong Avenue and Pudong South Road) has been started, and the transfer station of rail transit line 19 (planned) is set here. The west side is next to the Oriental Pearl TV Tower, and the main business area is business. There are 7 blocks in the study area, covering an area of about 44.9 hectares. The streets in this area are the main part of the main urban area of the city, carrying most of the employment, business and finance, economic and trade, cultural and leisure activities in Shanghai. A variety of transportation modes such as pedestrian, vehicle and rail transit are mixed and dense, and the quality of street space is worrying, which has high research value.

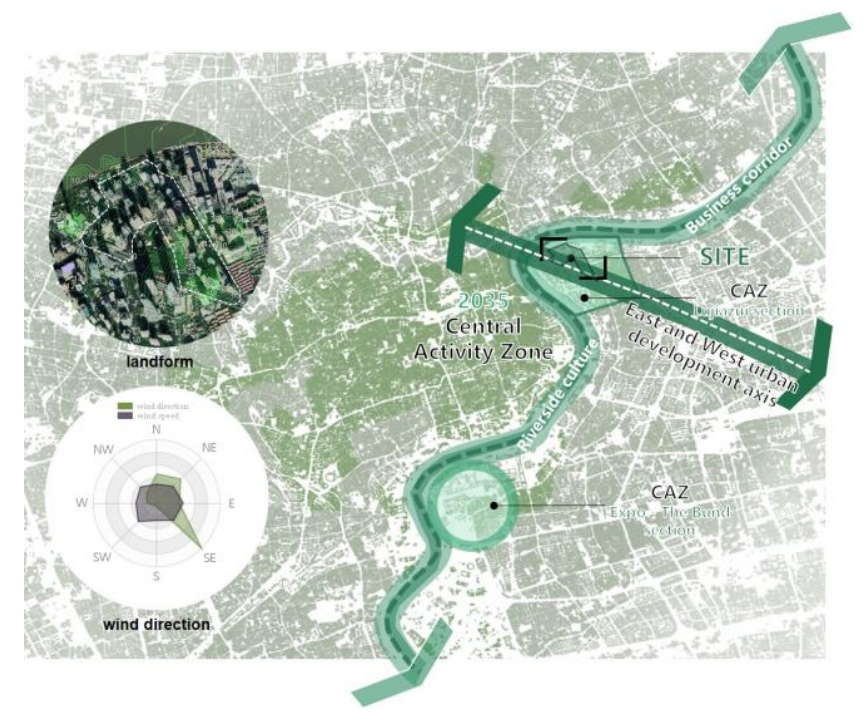

Figure 2. Research Scope. Source: Author. 


\subsection{Methods}

This paper summarizes the relevant research theories and practical methods by reading the literature in the field of street space quality at home and abroad; Based on street network shape data, open data of Internet comment platform and street view data of Internet map, taking geographic information system software ArcGIS as the main working platform, combined with Python programming software, Depthmap spatial syntax analysis software and SPSS statistical software for quantitative analysis of multi-source data; And use the method of qualitative analysis to evaluate the street space quality of Lujiazui area and explore its internal influence mechanism. Specifically, it includes the following research methods:

(1) Field research method

Conduct field research on Lujiazui area in Shanghai, mainly on each street in the research area, interview street users, observe and record the current situation and problems of street space.

(2) Big data analysis

Using the methods of Internet download and exchange and python programming code acquisition, this study obtained relevant open multi-source data such as vector map, map POI data, street view pictures, social media public comment data and crowd heat data of Lujiazui area in Shanghai, combined with semantic segmentation recognition tool based on in-depth learning and other corresponding analysis tools, Build a basic database based on Shanghai Lujiazui Street vitality analysis. In addition, the relevant data in the database are processed, the data are screened according to the research needs, and the data are visually analyzed, so as to lay a research foundation for subsequent analysis [17].

(3) Spatial analysis method

The spatial analysis method mainly analyzes the spatial distribution of crowd flow density and crowd aggregation distribution, the aggregation characteristics of map POI data and public comments by using the spatial analysis tools in ArcGIS software, and quantitatively analyzes the crowd heat, aggregation distribution and other data through spatial analysis, It can find out the vitality accumulation and vitality distribution characteristics of specific streets [18]; Analyze the aggregation characteristics of map POI data, get the activity behavior characteristics of street users on the street, classify them, and further do targeted research on vitality improvement. Through spatial analysis, multi-dimensional and multi angle targeted quantitative analysis of street vitality research, enrich vitality and improve research indicators, and lay a solid data and theoretical foundation for vitality quantitative research.

(4) Statistical analysis

The basic sample data of street spatial quality are further analyzed by using some analysis methods based on statistical principles, Excel, ArcGIS built-in analysis tools and other software, and the distribution characteristics and aggregation forms of activity elements carried out by street users in the street are quantitatively analyzed by using hot spot analysis, kernel density and other analysis methods, so as to summarize the proportion of different types of street activity elements, Then summarize the influence mechanism of street activity elements [19].

\subsection{Data Sources}

This paper shares 8 types of data: vector map, POI data, road network data, spatial form data, social media public comment data, crowd heat data, street view image data and business hours data along the street. Among them, vector map, POI data, road network data and spatial form data are crawled through OSM map; Crowd thermal data and street view image data are crawled through Baidu map open platform; Social media public comment data is obtained by crawling the evaluation information on public 
comments; The business hours of street businesses are obtained by consulting relevant network materials.

\section{Results}

\subsection{Street space capacity}

The indicators of street space carrying capacity include five factors: construction intensity, space form, street greening coverage, street height width ratio and street space identifiability. The construction intensity and spatial form of the study area are obtained by crawling the OSM data (Fig. 3). It can be seen that the development intensity of the study area is high, mainly high-rise and super high-rise buildings, and the plot ratio is greater than 5. From the relationship between the map and the bottom of the study area, it can be seen that in addition to the plots with high development intensity, there is also a large open space, which belongs to the pattern of wide opening and wide cooperation as a whole, which is in line with the image characteristics of the urban central area. In the analysis of street greening coverage (Fig. 4), it can be found that the ancillary green gardens, green belts, squares and other spaces in the study area are developed independently, with uneven quality and style, and lack of connection in space and style. At the same time, except for the Central Park, the street greening coverage rate in the study area is generally low and lack of green space. The street height width ratio (H/D) in the study area is similar, which is greater than 2 . The overall street space has a strong sense of oppression and the scale is not pleasant. Through the spatial syntax analysis, the street space recognizability evaluation is obtained (Fig. 5). It can be known that the higher the road grade, the better the vehicle permeability and accessibility. At the same time, due to the division of elevated roads, the pedestrian permeability and accessibility in the northeast and southwest of the study area are higher and lower. On the whole, the bearing capacity of street space in the study area is close to saturation, the development intensity is high, there is a lack of open space such as green space, and the pedestrian suitability is poor.

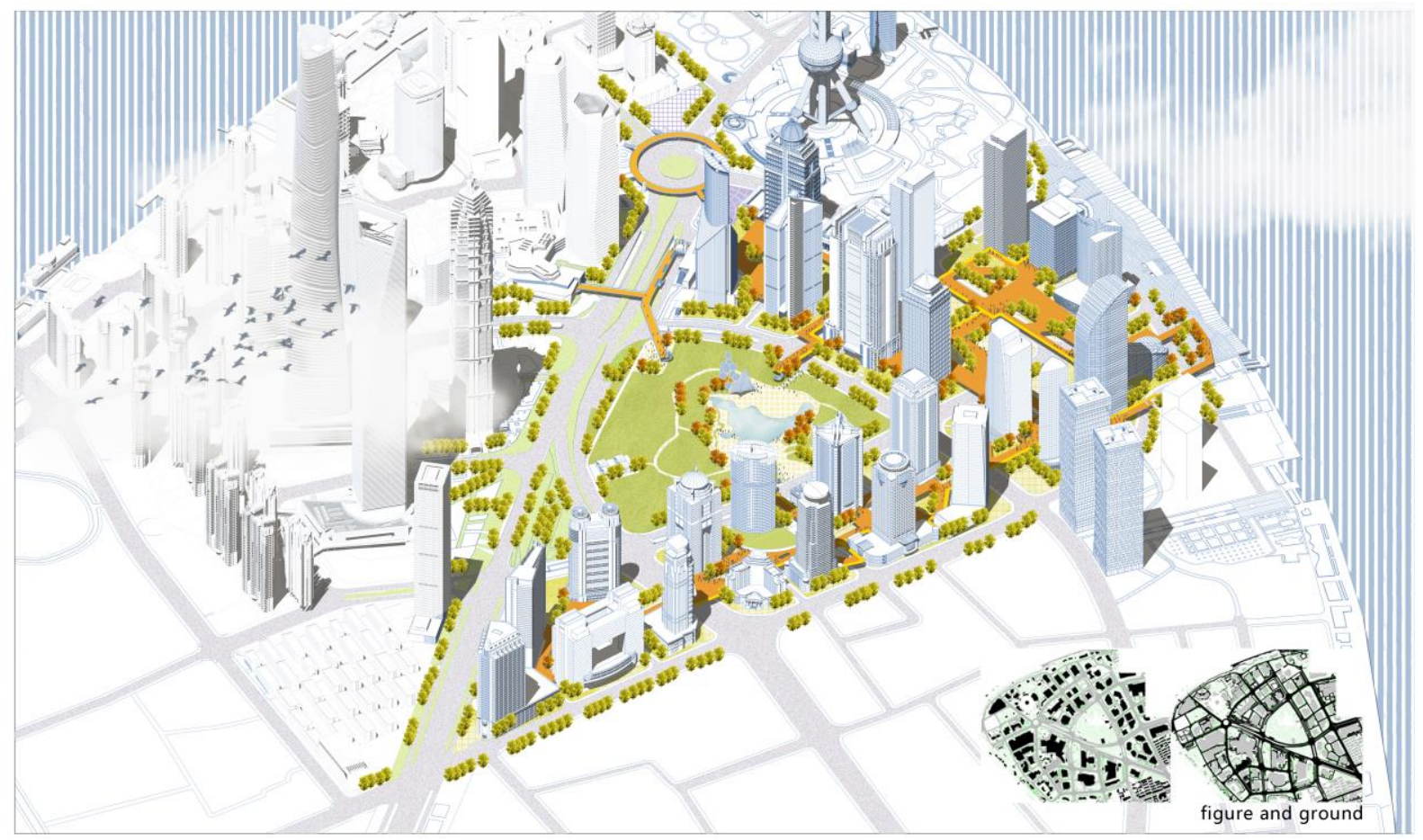

Figure 3. Construction intensity and spatial form analysis. Source: Author. 

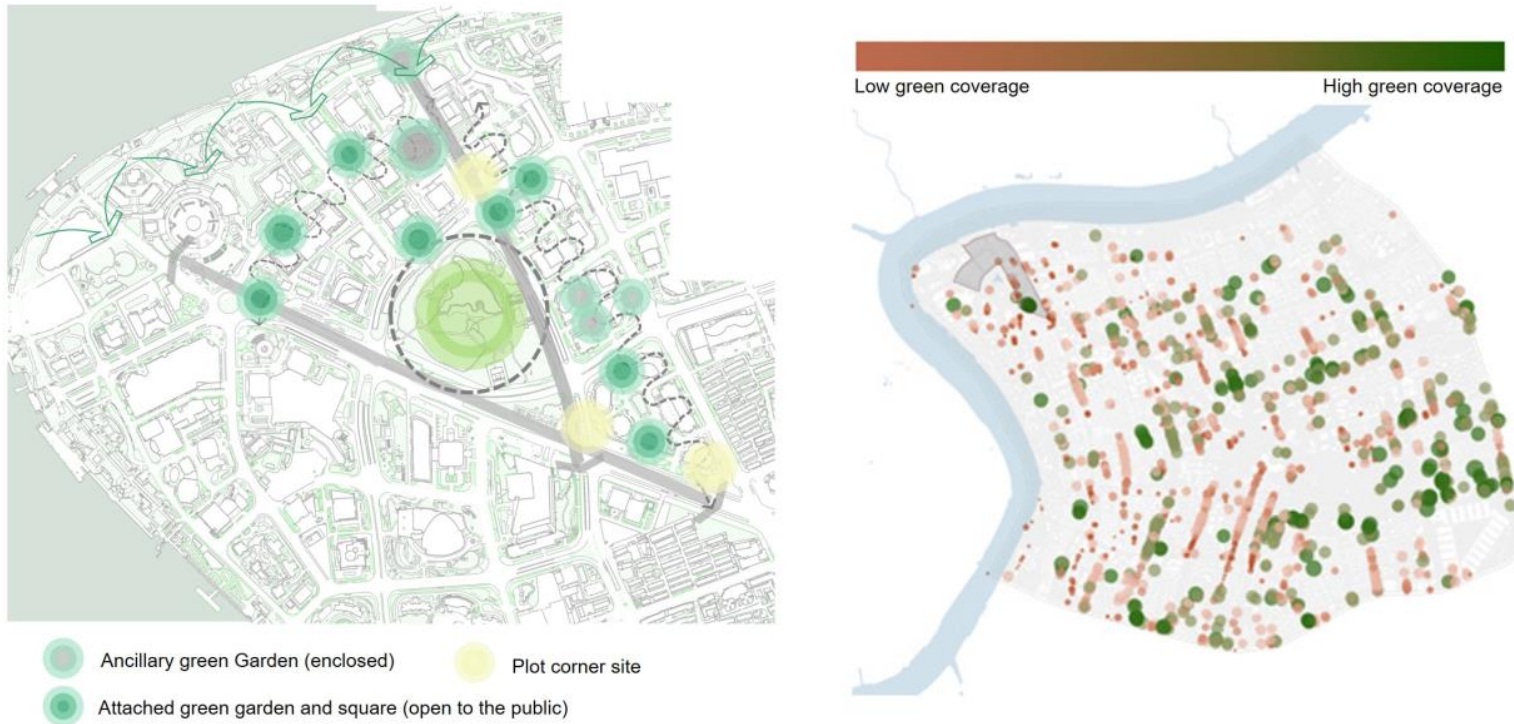

Figure 4. Analysis of street greening coverage rate. Source: Author.

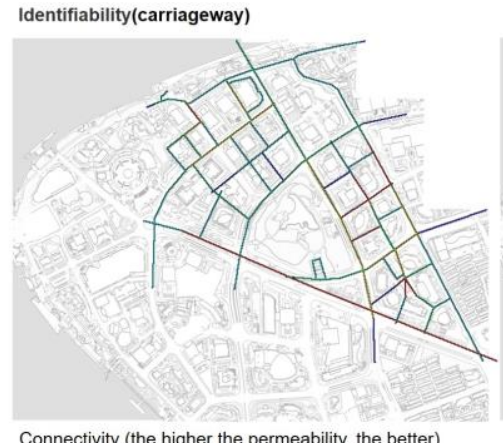

Connectivity (the higher the permeability, the better)

Identifiability(sidewalk)

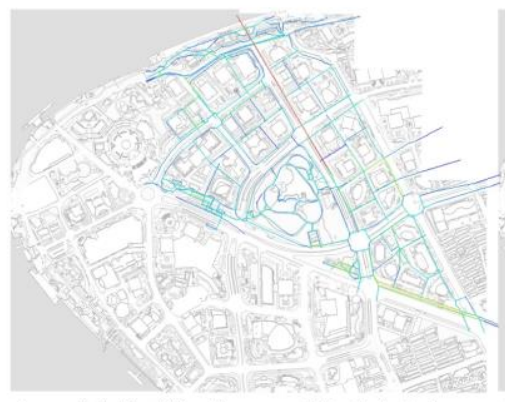

Connectivity (the higher the permeability, the better)

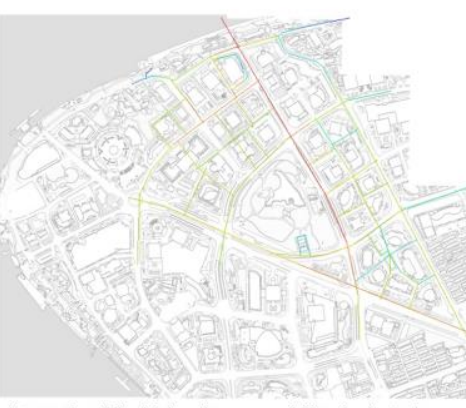

Integration (The higher the accessibility, the better)

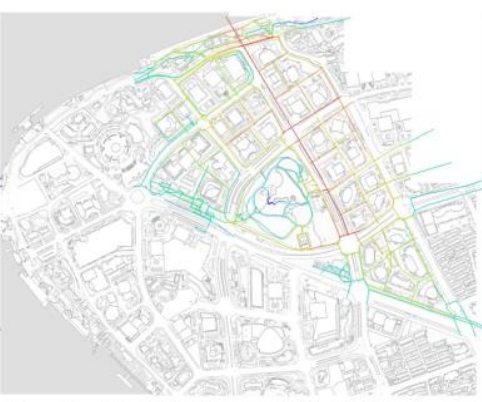

Integration (The higher the accessibility, the better)

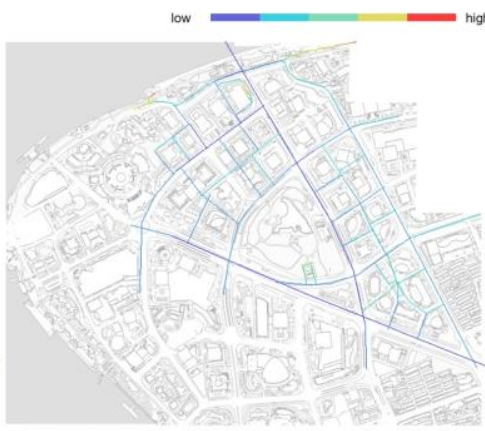

Depth value (the lower the accessibility, the better)
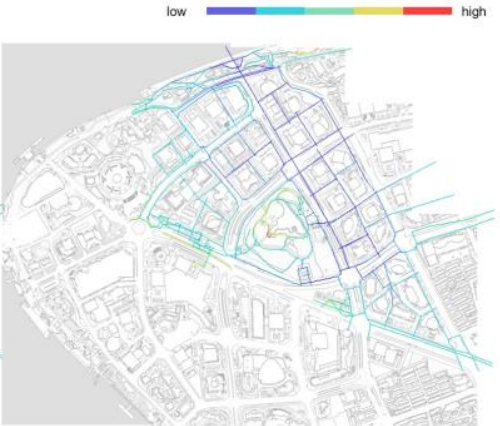

Depth value (the lower the accessibility, the better)

Figure 5. Analysis of street space identification. Source: Author.

\subsection{Street Space Vitality}

Street space vitality indicators include four factors: Street function, street scene type analysis, street facade color analysis and street business hours analysis. By crawling the POI data of various business types in the study area through Baidu map and analyzing the street functions by using ArcGIS software (Fig. 6), it can be found that the functions of accommodation services and shopping facilities in the study area are extremely scarce, and the functions of business houses and scenic spots are also relatively scarce, including financial insurance, transportation facilities, companies Public facilities and other functions are rich and concentrated in the southwest of the study area, and the stock of other facilities is medium. 
Based on the street scene type analysis of Baidu map database (Figure 7), the street types in the study area are divided into 6 categories. It can be found that there are more traffic oriented streets and less green roads and pedestrian or activity space in the study area. Most of the buildings on both sides of the street are modern style buildings, and glass curtain walls are used more. The colors inside are mainly white, gray, light yellow and other elegant colors. According to the analysis of business hours of street businesses (Fig. 8), most of the business hours of the streets in the study area are 7:00-22:00 (the office is open 24 hours). The higher the street level, the longer the business hours. On the whole, the vitality of street space in the study area is poor. On the one hand, due to the single function of the street and the service nature of bringing more people, there are fewer functions and shorter business hours; On the other hand, the streets bear more traffic functions, which weakens the openness and sharing of the streets to people to a certain extent, resulting in the lack of vitality of the streets.

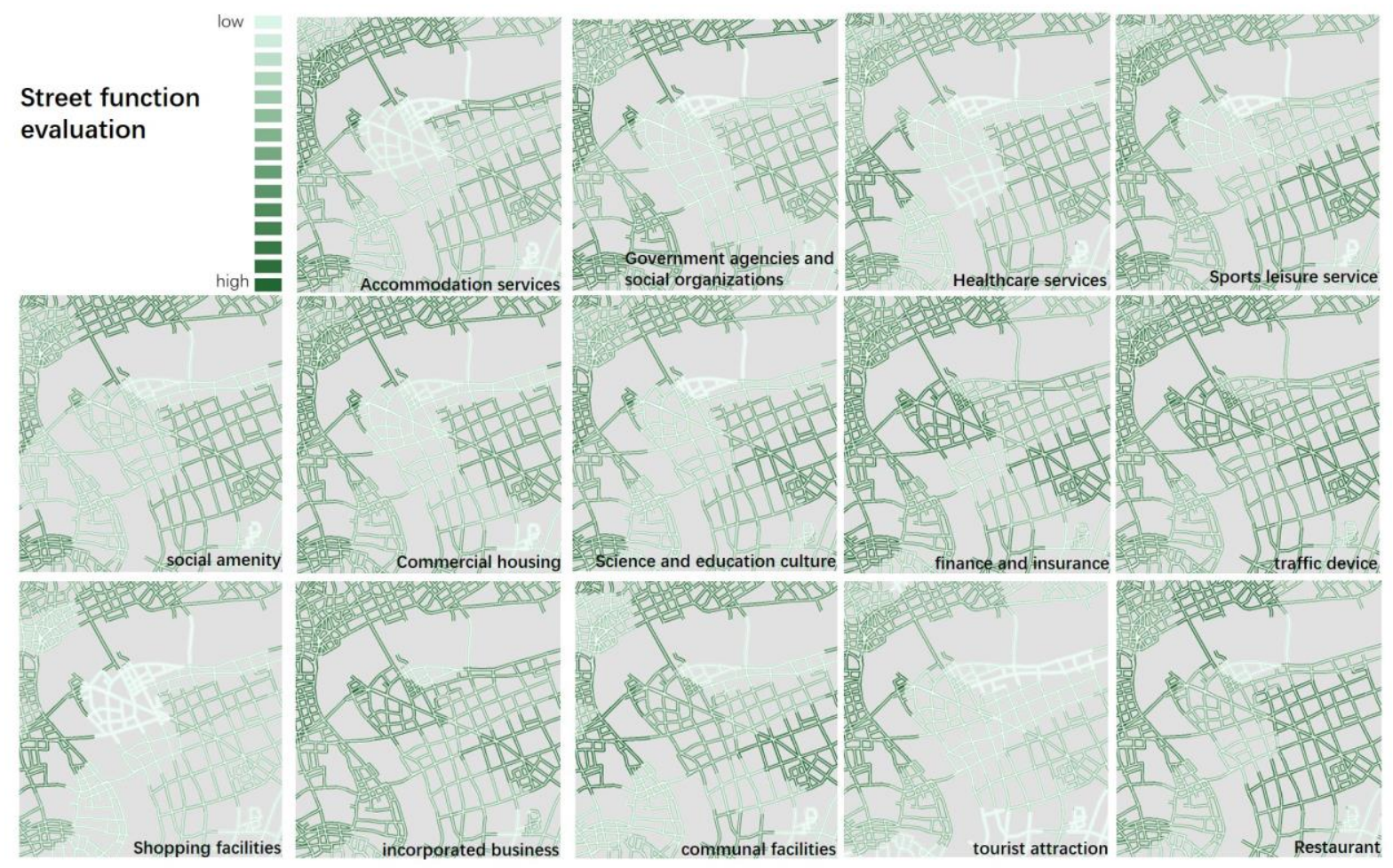

Figure 6. Street function analysis. Source: Author.

\subsection{Comfort of street environment}

The comfort index of street environment includes three factors: walking convenience, landscape beauty and facility perfection. By selecting several representative points in the study area for horizon analysis and field investigation and analysis (Fig. 9), it can be seen that there are areas with small horizon in the study area. At the same time, field investigation and analysis show that the landscape on both sides of the street is less beautiful, mostly small strip green space, small scale and low quality. The semantic segmentation recognition tool based on deep learning and other corresponding analysis tools get the panoramic segmentation analysis of street view image (FIG. 10). Combined with the field interview, it can be found that there are more motor vehicles, fewer bicycles and people, and poor walking convenience in the study area; Combined with the above street function analysis results, it can be seen that some functions of the street are insufficient, the number of street furniture is small, and the perfection of street facilities is low. On the whole, the pedestrian friendliness of the street is low, mainly by car, the landscape is broken, there is no connection, the overall aesthetics is poor, and the facilities are imperfect, so the comfort of the street environment is poor. 

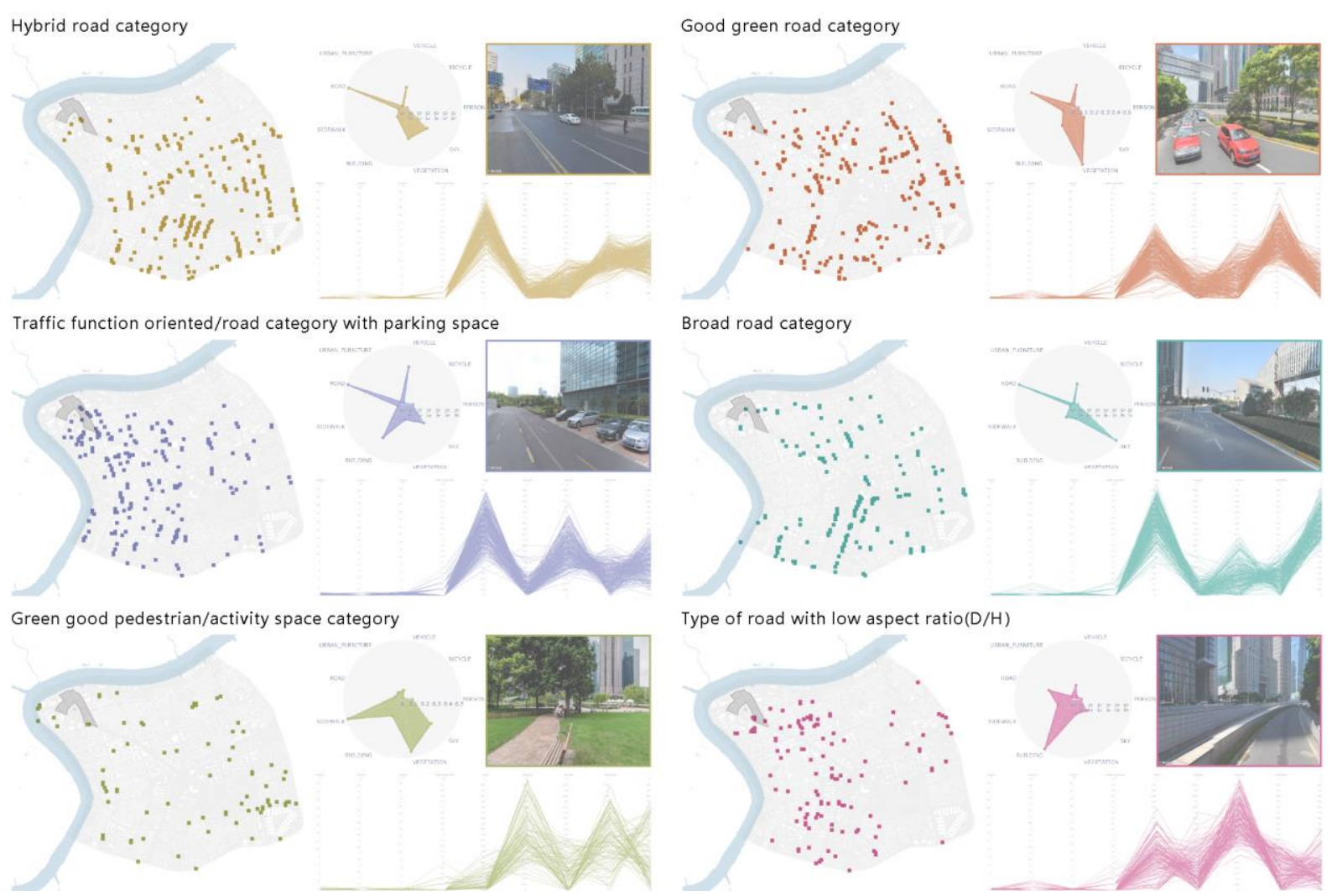

Broad road category

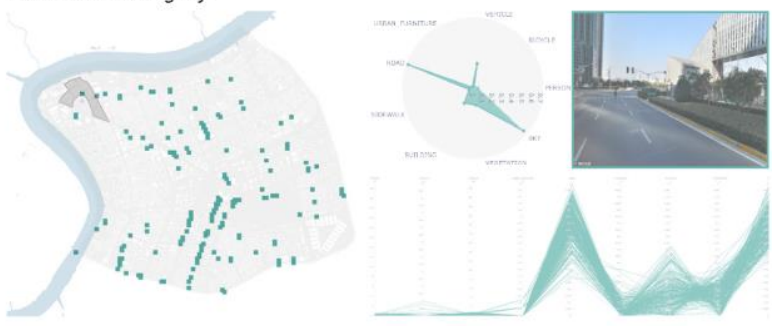

Type of road with low aspect ratio(D/H)

Figure 7. Street scene type analysis. Source: Author.
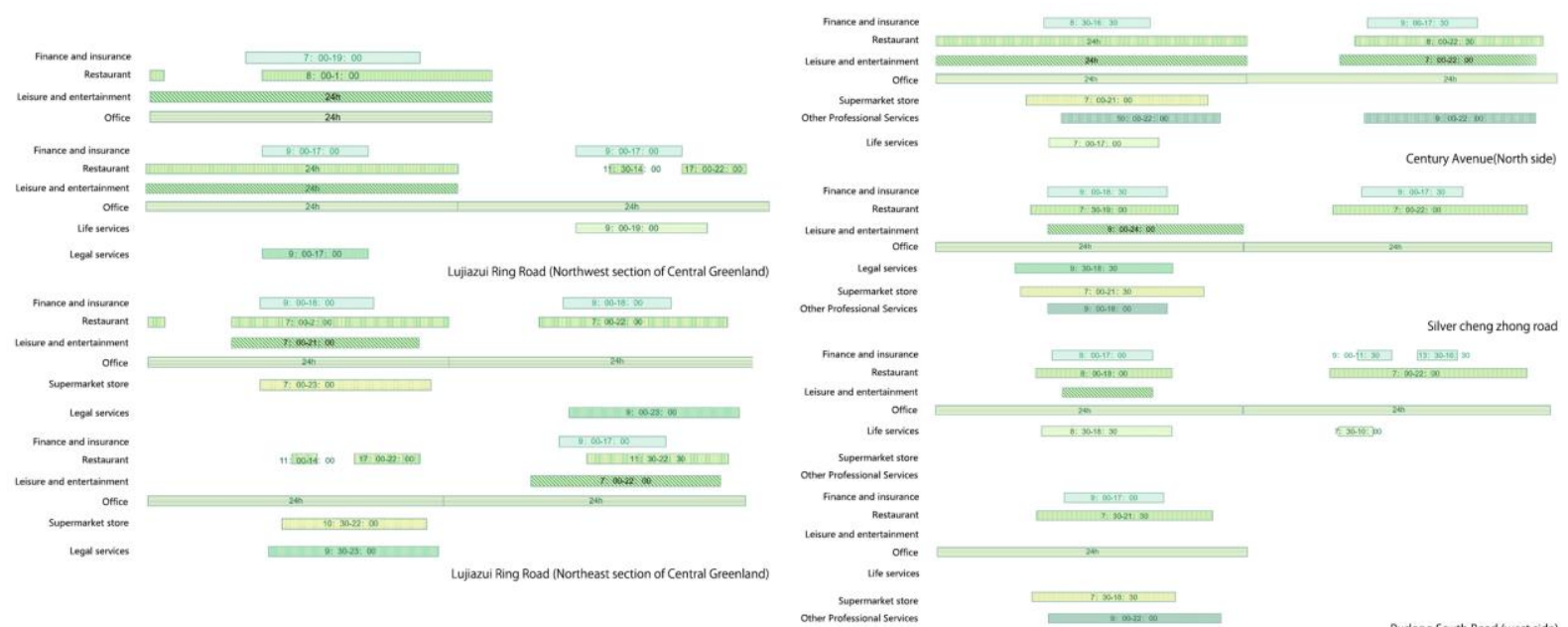

Figure 8. Analysis of the business time of street format. Source: Author.

\subsection{Street travel safety}

Street travel safety indicators include street brightness, vehicle interference index and the perfection of marking facilities. Combined with the results of panoramic segmentation analysis of street view image above (FIG. 10), except for the road intersection close to the Central Park, the percentage of street sky area is generally low within the research scope. It can be seen that due to high-intensity development, the brightness of the street is poor, which needs to be supplemented by lighting. At the same time, the study area is mixed with pedestrians and vehicles, with a large number of vehicles and serious vehicle interference. Through field investigation, it is found that the marking facilities in the study area are relatively perfect, but due to the collection of various traffic modes, the marking system is chaotic and 
not eye-catching enough. In particular, non motor vehicles are prohibited from entering on some roads. Due to the unclear marking, there are many false entrances, affecting the normal operation of street traffic. Overall, the evaluation of these three factors is poor, so the street travel safety is poor.
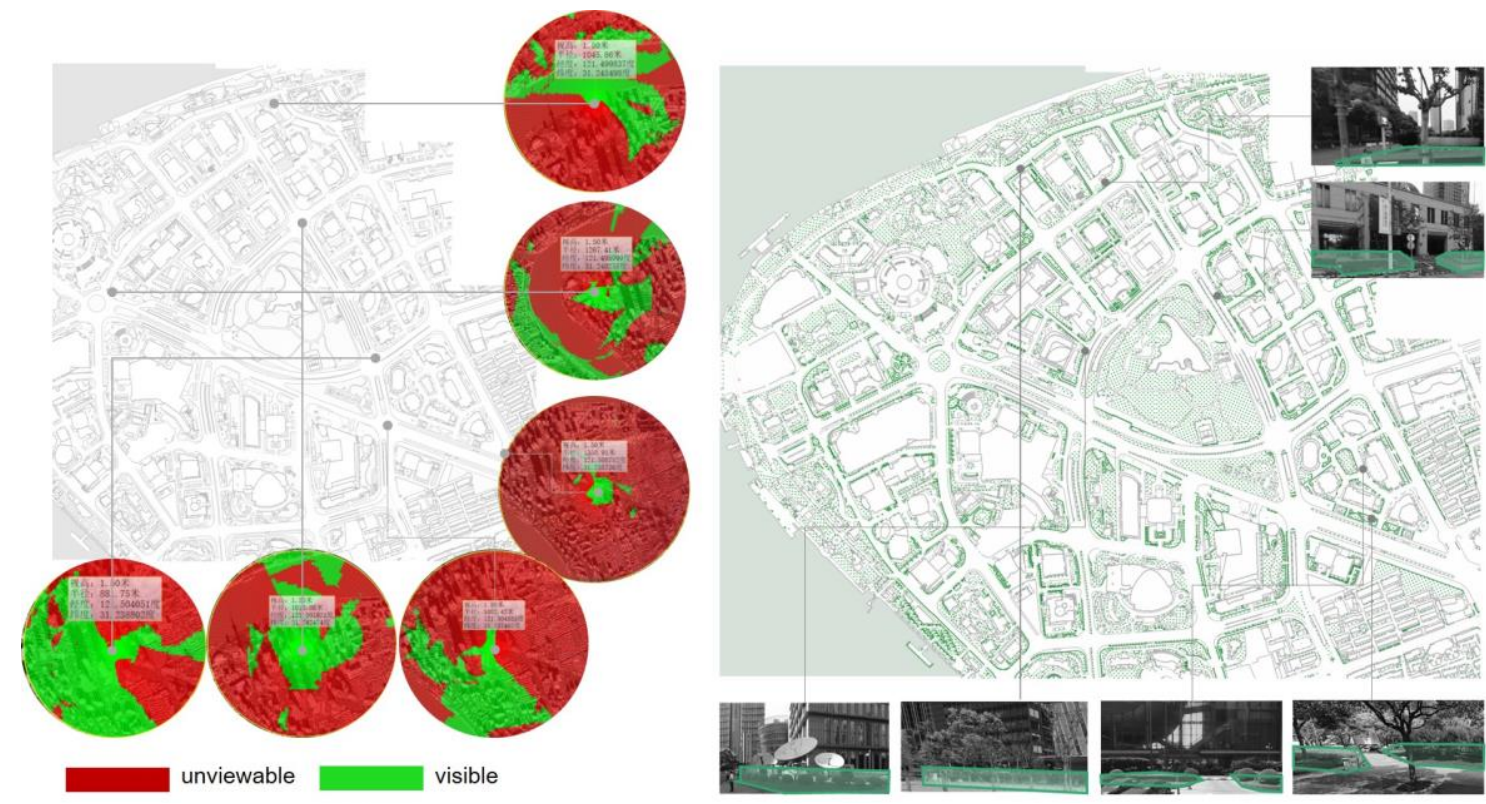

Figure 9. Aesthetic degree of landscape. Source: Author.
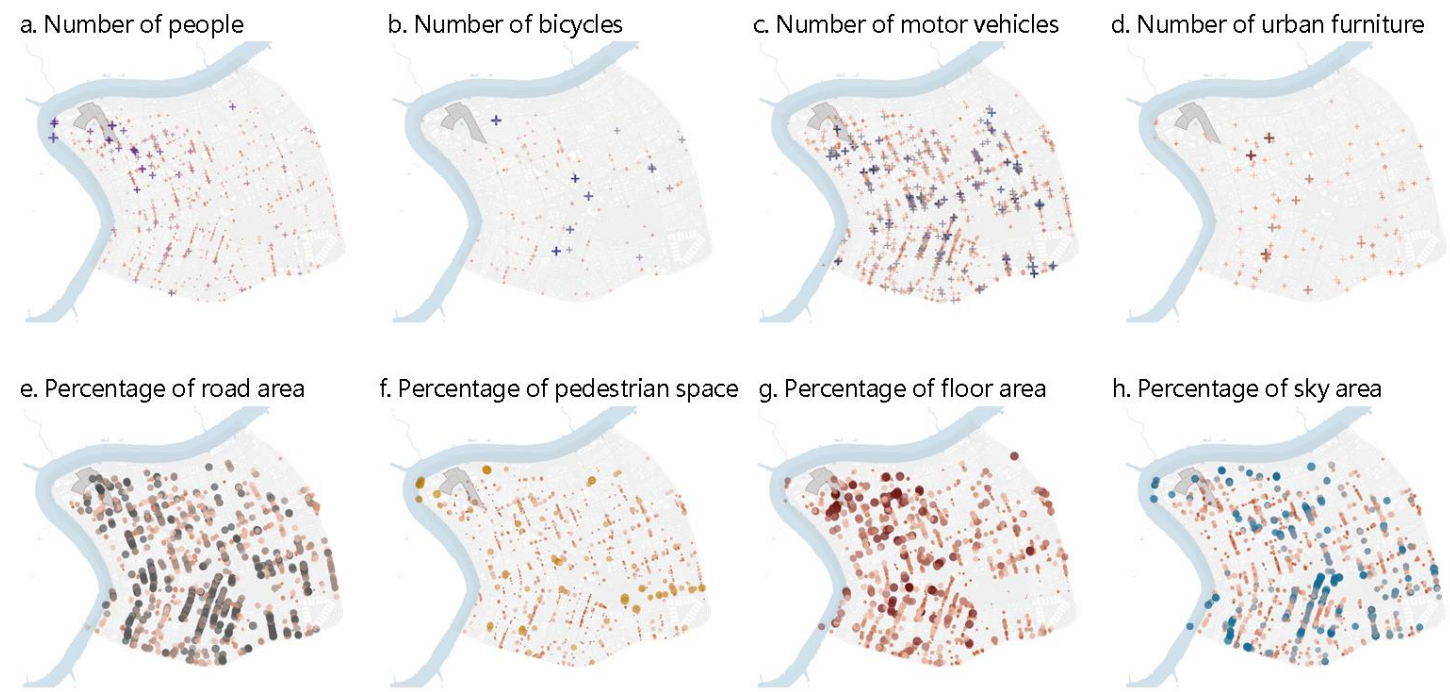

h. Percentage of sky area

Figure 10. Panoramic segmentation analysis of street view image. Source: Author.

\subsection{Crowd social interaction}

The social interaction index of the crowd includes three factors: Baidu heat map, crowd agglomeration degree and social interface index. Through the analysis of Baidu thermal map and crowd agglomeration degree (Figure 11), it can be seen that crowd activities are mainly concentrated in the period of 12:0020:00, and there are few people in the period of 20:00-24:00. It can be seen that there are a large number of commuters. By crawling the evaluation data from the public comments for keyword analysis, the urban environmental emotion analysis (Fig. 12) is obtained to evaluate the street social interface index. It can be found that there are more disgust and sadness in the study area, reflecting that the dissatisfaction of street users with street space is the mainstream. On the whole, the crowd activity time is concentrated, the space satisfaction is low, and the crowd social interaction is poor. 
Concentration of Pedestrian Flow along the Main Roads
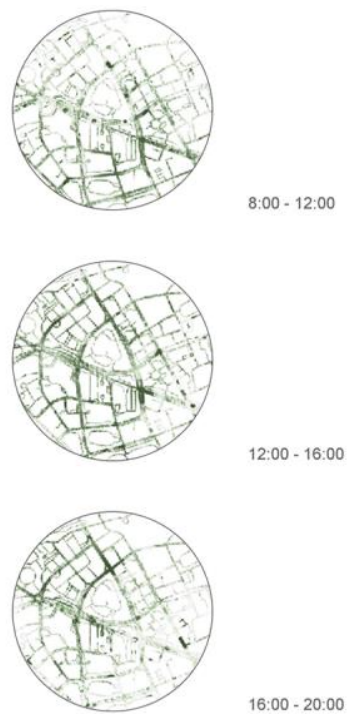

$16: 00-20: 00$

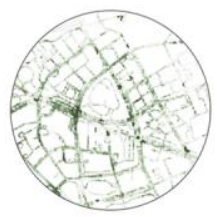

$20: 00 \cdot 24: 00$
Distribution Density the Population in Buildings

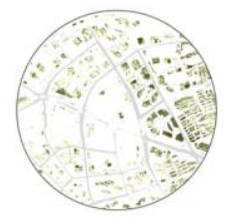

$8: 00-12: 00$

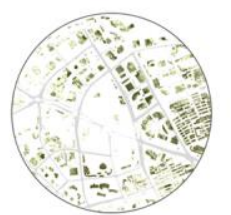

$12: 00-16: 00$

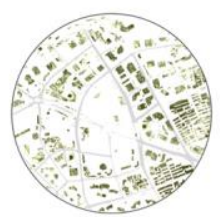

$16: 00-20: 00$

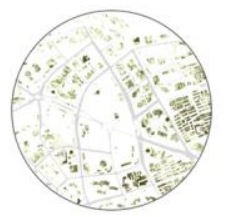

Congregation of the Populatic Based on LBS Data Points

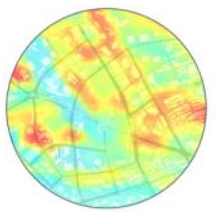

$8 \cdot 00-12 \cdot 00$

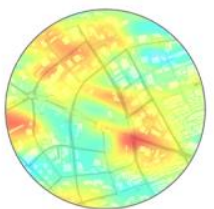

$12: 00-16: 0$

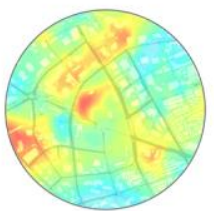

$16: 00-20: 06$

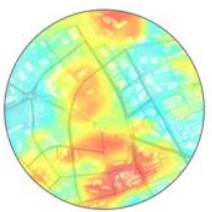

Figure 11. Baidu thermal map and crowd aggregation degree analysis. Source: Author.

\begin{abstract}
Heat map based on Street View sentiment - disgust
\end{abstract}

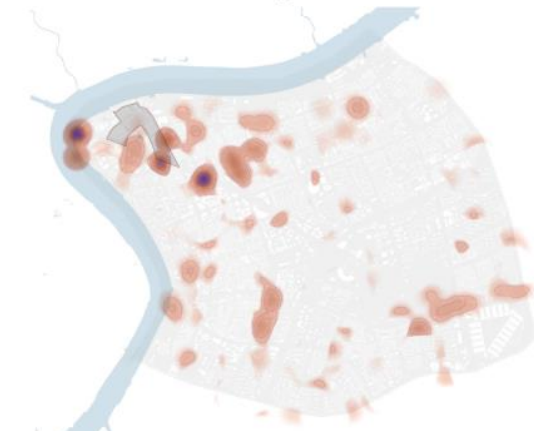

Heat map based on Street View sentiment - sadness

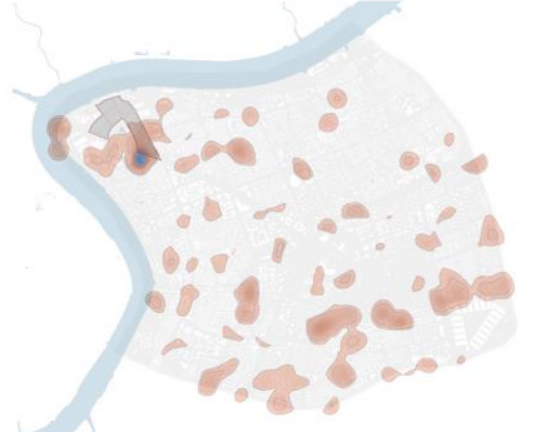

Heat map based on Street View sentiment - fear

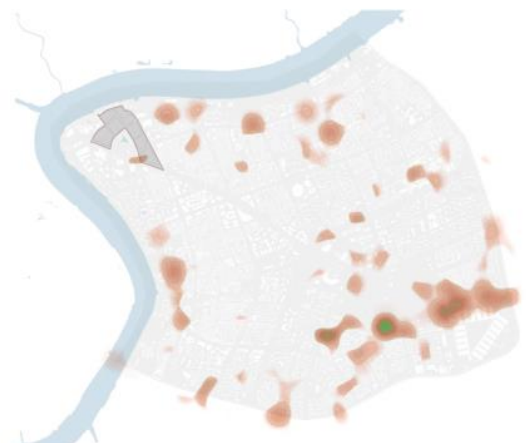

Heat map based on Street View sentiment - happiness

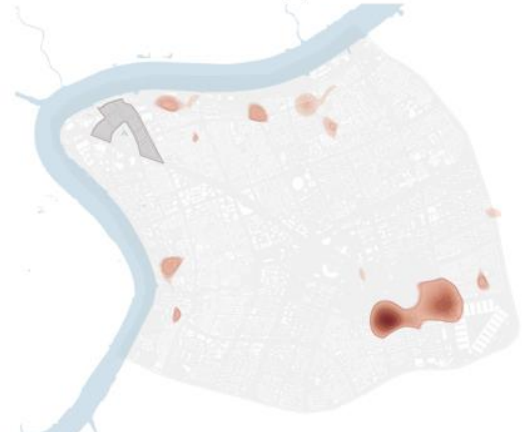

Figure 12. Urban environmental sentiment analysis. Source: Author. 


\section{Discussion}

Through the above evaluation and analysis, it is found that there are mainly the following problems in the study area: first, the function of the public space in the block is single, and there is a lack of positive street interface. The current situation is mainly people, vehicle traffic and parking, and there is a lack of the function of providing services for people or adding regional vitality. Second, the slow space between blocks is discontinuous and interrupted by urban roads; The slow-moving space in the block is of poor quality and is mostly occupied by road parking. Third, there are some imperfect public facilities in the street space. Fourth, it has the characteristics of a large number of stakeholders involved in urban renewal. In view of the above problems, based on the study of the attributes of regional business blocks, this paper puts forward promotion strategies from three aspects: street public space, street public facilities and street support system.

\subsection{Street public space}

(1) Enrich Street functions and optimize the interface of buildings along the street

Pay attention to the humanized use needs of the streets and improve the functional diversity and vitality of the streets in the business district. Add recreational and leisure services to the street space to stimulate the vitality of the street and attract people to stay. By reasonably controlling the height of buildings along the street and creating the style of buildings along the street, we can create a positive interface of the street and meet the diversified space needs of people, such as comfort, convenience and safety.

(2) Improve the continuity and quality of street slow traffic space

Improve the continuity and quality of slow traffic space in the Business District neighborhood, and carry out slow traffic space design. According to the use needs and activity experience needs of various users in public space, humanized Street scale and good slow travel experience are formed through slow travel space design.

(3) Optimize street greening landscape

Greening along the street can enrich the landscape level of the street, enhance the visual beauty of pedestrians, enhance the vitality of the street and activate the street atmosphere. Based on the regional characteristics of the street, try to use local green plants as the basis for greening along the street. In addition to using flower beds to arrange greening along the street, green plants can also be combined with buildings along the street, such as using the outer wall of buildings along the street to arrange greening. At the same time, the corner space can be used to transform the micro garden, break the original space boundary, open the space to pedestrians, and enrich the space content by setting landscape sketches, shadow Gallery, children's activity space and so on.

\subsection{Street public facilities}

(1) Make up the short board of street public facilities

The number of street public facilities is large and the types are complex. Generally, they can be divided into two categories, namely, indication and sign public facilities and urban furniture public facilities. According to the current problems and the needs of users in the business district, public space and existing buildings can be used to supplement the short board of street public facilities, so as to provide convenient, diverse and efficient service function support for users in the business district, so as to add regional vitality and catalyze the development of surrounding areas.

(2) Optimize and improve the quality of public facilities 
The optimization of street public facilities is based on intensive sharing and enhanced interaction. On the one hand, the setting of public facilities should be energy-saving and environmental protection as much as possible to avoid resource waste. On the other hand, the interaction of public facilities can be enhanced through activities to create a beautiful, clean, hygienic, creative and interesting street environment. At the same time, the function of barrier free traffic safety should also be taken into account.

\subsection{Street support system}

(1) Establish multi-agent update mechanism

The renewal and reconstruction of street space often involves multiple subjects. The renewal mechanism can be explored by means of case reference and renewal mechanism design, and a highly operable multiagent renewal mechanism can be established to help the better implementation and follow-up maintenance of street space transformation.

(2) Explore smart block planning

Explore the methods of applying existing technologies such as big data and artificial intelligence to the function improvement of street space, slow space design or update mechanism design. On the one hand, provide more comfortable and convenient facilities and services to street users; On the other hand, it provides more intelligent and sustainable operation support for neighborhood management and designers.

\section{Landscape activity space optimization}

Activate corner space

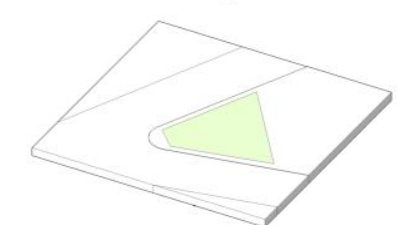

Unified green space design

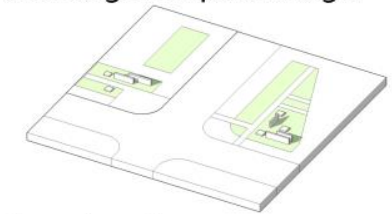

Boundary zigzag

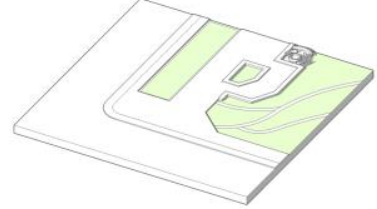

Get through the border

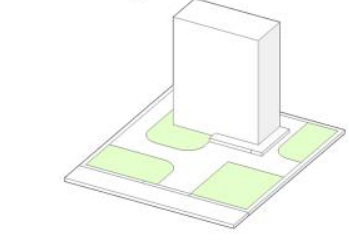

Rich content

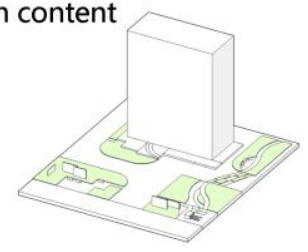

\section{Spatial content library}
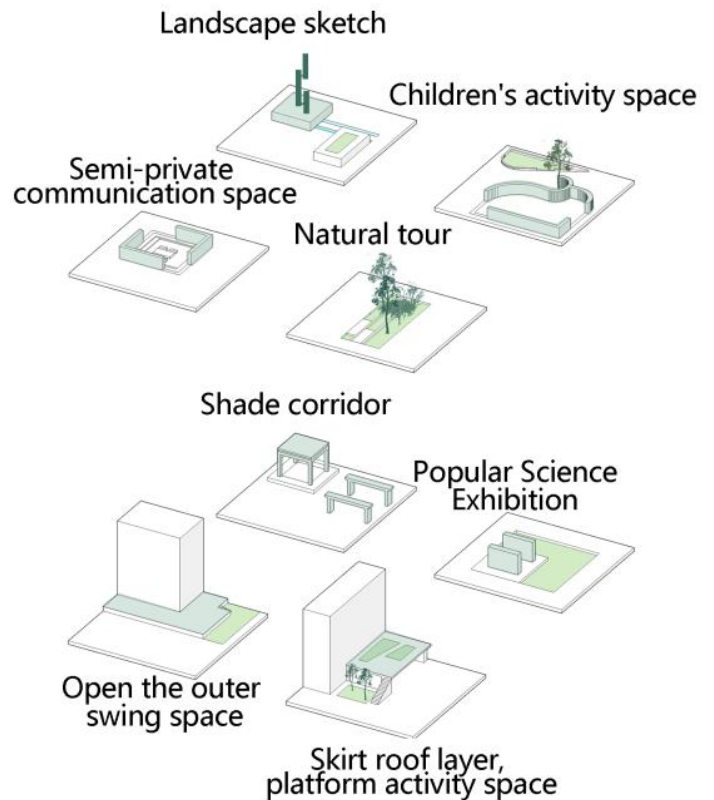

Figure 13. Street public space enhancement strategy. Source: Author.

\section{Conclusions}

By constructing the street space quality evaluation system, this paper evaluates and analyzes the street space in the core area of Lujiazui, Shanghai. The results show that its various service formats are low in richness, the slow travel system is broken, the street humanization degree is low, the street space lacks streamline and style connection, and the street service level does not match the high-density population 
This paper puts forward the improvement strategies from three aspects of the street support system, and points out that in the street planning and design, different types of squares and commercial facilities should be set up in combination with the diversified needs of residents, so as to make the street have diversified functions, improve the accessibility of traffic and the comfort of the street environment, so as to accumulate popularity and make the street more dynamic.

The evaluation system proposed in this study has low evaluation results for traffic trunk roads, but it fully meets the requirements as a functional road, so it is more suitable for the evaluation of living street space quality and has high universal applicability. The highlight of the research is to use multi-source big data and a variety of analysis methods to evaluate the street quality from the two dimensions of physical space characteristics and people's subjective perception. However, at the same time, due to the data sources and research technology, this study still has some limitations. In the follow-up research, a more comprehensive evaluation can be carried out by integrating the attributes of the street itself.

\section{References}

1. Moughtin C, (1992) Urban design: street and square. Architectural Press.

2. Whyte W F, (1943) Street corner society: The social structure of an Italian slum. Chicago:University of Chicago Press.

3. Jane Jacobs, (2006) Death and life of American big cities. Nanjing: Translation Press.

4. しはら よしのぶ, (2002) Aesthetics of streets. Jiangxi: Phoenix literature and Art Publishing House.

5. Jan Gail, (2010) Communication and space. Beijing: China Construction Industry Press.

6. Cliff Mumford, (2004) Streets and squares. Beijing: China Construction Industry Press.

7. Alan B. Jacobs, (2004) Great street. Beijing: China Construction Industry Press.

8. Cranshaw J,Schwartz R, Hong J L,et al. (2012)The Livelihoods Project: Utilizing Social Media to Understand The Dynamics of A City[C]//;City.58.

9. Jan Gehl, (2011) Life Between Building: Using Public Space. Island Press.

10. Biddulph M, (2012) Radical Street: The Impact of Innovative Street Design on Livability and Activity in Residential Areas. Urban Design International,17;178-205.

11. Reid Ewing, Clemente O, Neckerman K M, et al. (2016) Measuring Urban Design: Metrics for Livable places. Washington, DC: Islang Press.

12. Naik N, Philipoom J, Raskar R, et al. (2014) Streetscore: Predicting The Perceived Safety of One Million Streetscapes[C]//Computer Vision and Pattern Recognition Workshops (CVPRW), IEEE Conference on. IEEE, 2014:793-799.

13. Kendall, Michelle, Milsom, Johannes, Fraser Christophe, (2015) Epidemiological changes on the Isle of Wight after the launch of the NHS Test and Trace programme: a preliminary analysis. The Lancet Digital Health.

14. Sarkar C, Webster C, Pryor M, et al. (2015) Exploring Associations Between Urban Green, Street Design and Walking: Results from the Greater London Boroughs. Landscape and Urban Planning. The Lancet Digital Health.143:112-125.

15.Long Ying, Tang Jingxian, (2019) Research progress on large-scale quantitative measurement of urban street spatial quality. Urban planning,43 (06): 107-114. 
16. Ye Yu, Zhang Zhaoxi, Zhang Xiaohu, Zeng Wei, (2019) Measurement of street spatial quality on a humanistic scale -- a big study combining street view data and new analysis technology Scale and high precision evaluation framework. International urban planning,34 (01): 18-27.

17. Yang Junyan, Wu Hao, Zheng Yi, (2019) Study on spatial characteristics and optimization strategy of urban street walkability based on multi-source big data -- a case study of Nanjing Taking the downtown area as an example. International urban planning, (05): 33-42.

18. Wen Ning, (2019) Improvement and related application of spatial syntax axis analysis in urban street design. Tutor: Jia Xinfeng; Wang Ying Zheng State University.

19. Yang binyuan, Wei Haoyan, (2019) Study on walking friendly morphological characteristics of five streets in Milan based on three determinations analysis. Human settlements in Western China Journal, (01): 55-62. 